\title{
Non-signalized T-shaped Intersections Mixed Traffic Flow Study
}

\author{
Wenfang Feng \\ LanZhou University of Technology \\ School of Economics and Management \\ Lanzhou, China
}

\author{
Changsheng Zhu \\ Lanzhou University of Technology \\ School of Computer and Communication \\ Lanzhou, China
}

\author{
Yize Li* \\ Lanzhou University of Technology \\ School of Computer and Communication \\ Lanzhou, China \\ *Corresponding author
}

\begin{abstract}
Combining actual city T-shaped intersection traffic conditions in this paper, we propose a cellular automaton model of mixed traffic flow at T-shaped intersection. In order to avoid a collision between vehicles and gridlock phenomenon occurs, running vehicle in the model adopted the principle of major street vehicles traveling priority. In this paper, a simulation study in the open boundary conditions, three kinds of maximum speed of vehicles mix injected T-shaped intersection lane. Research branch street injection probability respectively impact $T$-shaped intersection of lane's average velocity and traffic flow. Under the same conditions, different mixing ratio coefficient of the speed are different effects on the traffic flow system. Main lane injection probability affect $T$-shaped intersection traffic flow within a certain range systems.
\end{abstract}

Keywords-cellular automaton model; T-shaped intersection; mixed traffic flow; injection probability

\section{INTRODUCTION}

In recent years, with the rapid development of urban transport, traffic congestion and traffic safety issues attendant worsening. Therefore, a reasonable transportation planning and management have become a key factor in a variety of traffic flow methods have been proposed. Among them, the use of cellular automaton theory (1-2) to study the traffic flow problems. This theory can make models of the evolution rules simply, easy to implement and simulate traffic flow micromovement state vehicle effectively, so it has been widely applied in the study of traffic flow in( 3-6 ).

Because the intersection of important position in the road network, the experts and scholars at home and abroad using cellular automaton model of traffic flow at the intersection of research (6-12). Tan et al (7) under open boundary conditions, the use of improved NS model, simulate traffic flow at the cross intersection of the city center of the major street and branch street. Xiao et al (8) under periodic boundary conditions, they researched the effects of vehicles' length, maximum speed, and the mixing coefficient ratio of traffic flow used by NS model. Zhang et al (9) studied of local non-interference mechanism intersections mixed traffic flow. Li et al (10) studied in the main lane to avoid a collision with two different regulatory simulate the non-signalized $\mathrm{T}$-shaped intersection of the major street and branch street traffic flow. Fan et al studied non-signalized T-shaped intersection main road vehicle injection probability, left-turning and right-turning traffic flow impact on intersection. $\mathrm{Li}$ et al non-signalized $\mathrm{T}$-shaped intersection in the role of the steering system of the vehicle and traffic systems to study the phase diagram.

However, domestic and foreign experts and scholars research characteristics of mixing traffic flow at $\mathrm{T}$-shaped intersection with multi-speed which is small based on cellular automaton model. Therefore, this paper established a consider all direction traffic direction T-shaped intersection with multispeed cellular automaton model. According to the actual nature exists in various types of vehicle, through different types of vehicles in the model with different maximum speed. This model simulated the running situation of non-signalized $\mathrm{T}$ shaped intersection is more close to actual. In this article, the model of the T-shaped intersection traffic flow characteristics were analyzed, the main research in a variety of maximum speed under mixed traffic flow conditions, branch lane injection probability of influence four lanes in T-shaped intersection system.

\section{MODEL AND EvOlution RULES}

\section{A. Cellular Automaton Model}

Non-signalized T-shaped intersection of cellular automaton model as shown in Figure I: the major street is consisted of Lane L3 and L2, the branch street is composed by lane L1. The major street and branch street vehicles are not allowed to overtake, in their respective lanes run independently. Each of the major street regarded as a discrete lattice chains the length of $L$, each of the branch street treat as a discrete lattice chains the length of $L / 2$. Every lattice point represents the length of $7.5 \mathrm{~m}$ in reality road, the status of every lattice point is empty(no car) or the vehicle which is occupied with speed of $V \in\left\{0,1,2, \ldots, V_{\max }\right\}$, here $V_{\max }$ is the maximum speed limit of vehicle can be achieved, the evolutionary step is $1 \mathrm{~s}(1)$. T- 
shaped intersection is composed of $\mathrm{C} 1, \mathrm{C} 2$, two cells. Only after the vehicle into the intersection $\mathrm{C} 1$ and $\mathrm{C} 2$, need to vehicle steering and driving into the branch L1. When the vehicle enters the intersection, there is a left-turning direction, right-turning direction and straight-driving direction traffic flow.

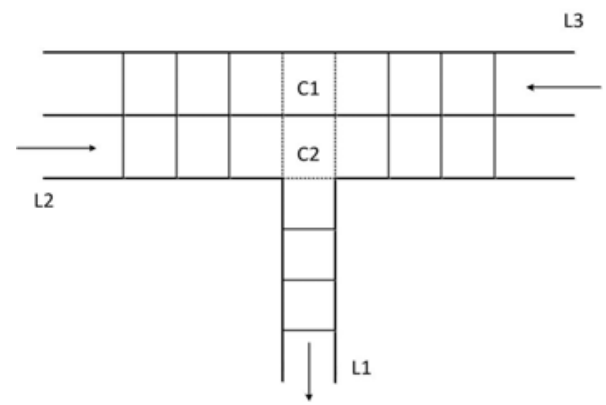

FIGURE I T-SHAPED INTERSECTION CELLULAR AUTOMATION MODEL

\section{B. Evolution Rules}

- In the boundary conditions, all of the major street and branch street's vehicles in accordance with NaSch rules (14) to be updated. NS model to achieve the vehicle acceleration and deceleration, randomization phenomenon. If mixed vehicles on non-signalized Tshaped intersection, they will update according to the four steps based on model as follows :

Step 1 Accleration: $V_{n} \rightarrow \min \left\{V_{n}+1, V_{\max }\right\}$

Step 2 Deceleration: $V_{n} \rightarrow \min \left\{V_{n}, d_{n}-1\right\}$

Step 3 Randomization: $V_{n} \rightarrow \max \left\{V_{n}-1,0\right\}$ with probability $\mathrm{p}$;

Step 4 Motion: $X_{n} \rightarrow X_{n}+V_{n}$

Here, ${ }^{n}$ and $X_{n}$ denote the speed and position of vehicle $n, d_{n}$ denote for the first time of $t$ and the car in front of $n$ close spacing between vehicles, $V_{\max }$ as the maximum speed of the vehicle. And the provisions of the opposite direction of vehicles are not allowed to car overtaking. When the vehicle entering the intersection to consider three different directions vehicles: the straight-driving, left-turning, right-turning vehicles, however straight-driving vehicles are provide with priority rights. When left-turning's vehicles come across rightturning's vehicles in the T-shaped intersection, make rightturning's vehicles go ahead.

1) The Rules of Avoiding Conflict at the Intersection: Under controlled conditions in the absence of signalized lights, in order to avoid collision among vehicles, into each lane at the intersection has a different priority. a) First of all, if the conflict (automata (C2) has been dominated by a car, turn left to the vehicle will stop in cell $\mathrm{C} 1$, waiting to meet after the operation rules in turn into the L1.

b) After the vehicle system into the intersection, if the conflict (automata (C2) is not occupied by a vehicle, first arrived in the conflict (automata $(\mathrm{C} 2)$ of the vehicle with the exercise of right of priority.

c) If the vehicle reaches the conflict phase at the same time, the time needed for the main road straight vehicles with the exercise of right of priority.

d) The vehicle according to the probability and speed of mixing ratio of injected L3 and L2 into the lane highway entrance, parallel update and system of the vehicle in accordance with the rules of the evolution of the position and speed.

e) If you enter the system in the vehicle motion, remove the car. Each time step of vehicle will be removed from the system loss probability.

Only vehicles meet the above conditions, it can be normal driving system in the T-shaped intersection. If will enter the intersection vehicles that dissatisfy the above conditions, then need to wait at the intersection until content the traffic state to enter.

\section{NUMERICAL SIMULATION RESULTS AND ANALYSIS}

The ith lane is divided into a certain numbers of sites and their number indicated as $L$, the number of vehicles on the lane at $t$ time is $N_{i}(t)$, and the density pand the average velocity $V$, the flow $J$ show respectively:

$$
\begin{gathered}
\bar{V}(t)=\frac{1}{N(t)} \sum_{i=1}^{N(t)} V_{i}(t) \\
\rho(t)=\frac{N(t)}{L} \\
J_{i}=\bar{V}^{*} \rho
\end{gathered}
$$

In T-shaped intersection simulation, the major lane L3 and L2 length of 1023 cells, the branch lane L1 length of 512 cells. C1 (C2) is located in 512 cell. Each cell corresponds to the actual road length $7.5 \mathrm{~m}$, an evolutionary step for the $1 \mathrm{~s}$. Set the maximum speed limit $V_{\max }=\{2,3,5\}$, the speed ratio coefficient $f_{2}, f_{3}, f_{5}$. Set up the system model of the vehicle of the injection can be divided into three types: fast car the maximum limit speed of the $V_{\max }=5$ cell/s, medium speed car maximum limit speed of $V_{\max }=3$ cell/s, slow car maximum limit speed of $V_{\max }=2$ cell/s. Each sample runs 
50,000 time steps, the average of the numerical results versus time is taken from the last 10,000 time steps, and 20 samples are taken for the assembly average to eliminate the influence of randomness. Taking into account the influence of branch lane injection probability of each lane change into the average speed, density, traffic flow. Set the main lane injection probabily $a_{2}=a_{3}=0.7$, disappear probability $\beta_{2}=\beta_{3}=1$, turning probability $P_{2}=P_{3}=0.3$, disappear probability $\beta_{1}=1$, randomization probability $p=0.2$. Mixing ratio shall meet the following conditions:

$$
{ }_{0 \delta} f_{2}, f_{3}, f_{5} \delta 1, f_{2+} f_{3} f_{5}=1
$$

\section{A. The Main Injection Probability Affect of Mixed Traffic Flow Lane Average Speed}

Figure II respectively in different speed mixing ratio condition, the relationship between the main road injection probability and L2, L3, L1 on the average speed of the vehicle lane. From the Figure II can be drawn: under the same conditions (same injection probability, diverted same probability, the same random slow probability, the same exit probability), the greater the mixing ratio of the coefficients of fast cars proportion in each lane the average speed of the vehicle increases. From Figure II (a), (b) two figures can be seen, under the same conditions, L2 lanes are slightly larger than the average speed of vehicles on the average speed of the vehicle lane L3. The T-shaped simulation results accord with the intersection of L2 in the system on the right turn vehicle lane set prior to the L1 Lane on the left turning vehicles entering the road L1. Under the condition of mixed proportion coefficient is $f_{5}: f_{3}: f_{2}=1: 0: 0$ in speed, in Figure II (a), (b) found: when the main road injection probability $a_{2}<0.45$ the average speed of the main vehicle in maximum and there is no too big fluctuations; In Figure $\mathrm{II}(\mathrm{C})$, the injection probability changing process from 0.025 to 1.0 , the curve smooth throughout, the average speed of vehicles on the road remains substantially unchanged.

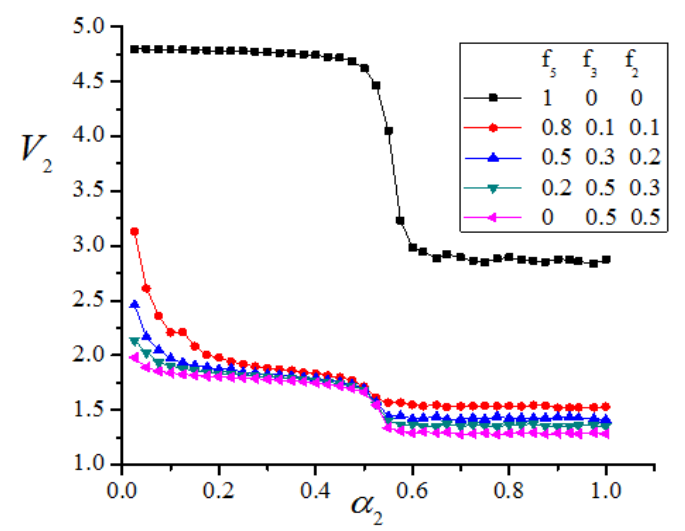

(a)

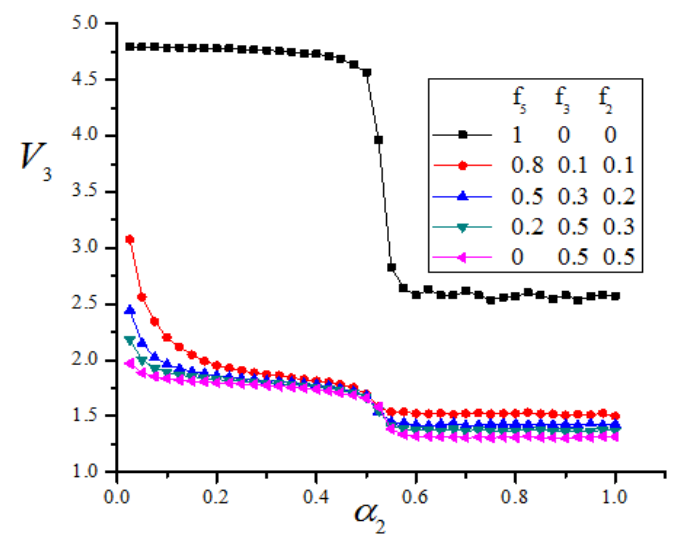

(b)

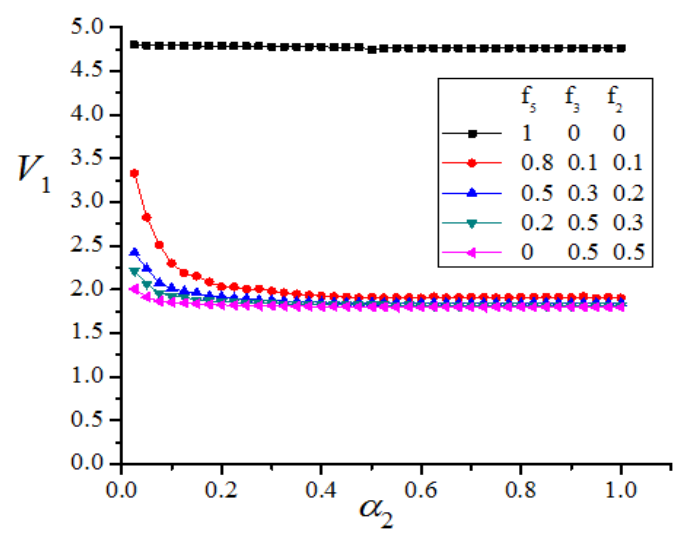

(c)

FIGURE II THE RELATIONS DIAGRAM BETWEEN THE MAIN LANE INJECTION PROBABILITY AND THE LANE AVERAGE SPEED

In the other four speed mixing ratio factor conditions, the same trend curve. Figure II (a), (b) the main road when injected probability $a_{2}<0.55$, the average speed of vehicles on the roads decreases. When the main road injection probability $\left(a_{2}>0.55\right)$, continues to increase, the injection probability no longer impact on the average speed of vehicles on the main road. Figure II (c), when $a_{2}<0.15$ the average speed at the time the branch road L1 vehicle gradually decreases. When injection probability of continuing to increase, will not be supported on the average speed of vehicles affected lane.

\section{B. The Main Injection Probability Affect of Mixed Traffic Lane Flow}

Figure III is under the condition of different speed mixing proportion, the relationship between branch injection probability and T-shaped intersection system of three lanes' traffic flow. From the Figure II can be drawn: under the same conditions (same injection probability, diverted same probability, the same random slow probability, the same exit probability), the greater the mixing ratio coefficients of fast cars proportion in each lane the traffic flow increase. The other four mixing ratio coefficient conditions, changes in the flow of the same trend, value or less. Show that a variety of other speed mixing ratio of each lane traffic impact is relatively small. 
From Figure III(a), (b) the results: under the same conditions, on the main road L2 flow slightly greater than the L3 traffic flow. Analysis of Figure III(a), (b) the data found: when branch road injection probability, the curve substantially coincide, the speed will not affect the mixing ratio of the main road traffic flow. Analysis Figure III (c) of the data found that: When injected Road branch probability ( $a_{2} \leq 0.2$ ) is small enough, the curve essentially coincident, indicating that speed does not affect the branch lane L1flow of the mixing ratio .In Figure III(a), (b), (c) be drawn: When the branch lane injection probability ( $a_{2}<0.55$ ), three lanes' traffic flow are increasing. With the support to continue to increase injection probability ( $a_{2}<0.55$ ), curve leveled off, indicating that the traffic lane remains unchanged.

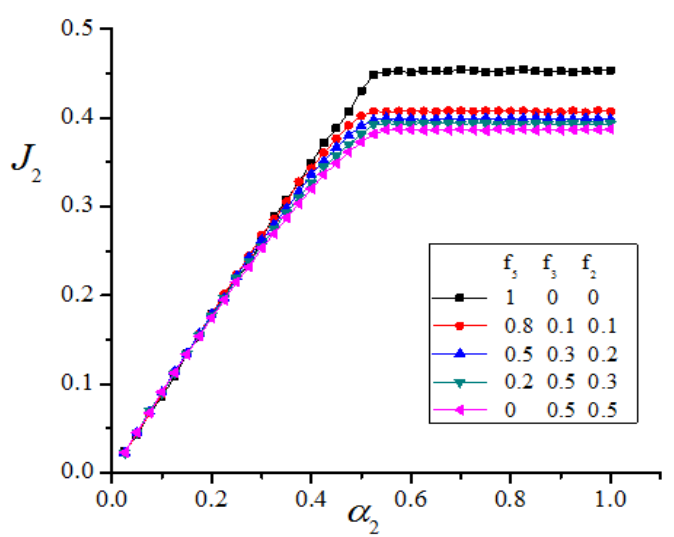

(a)

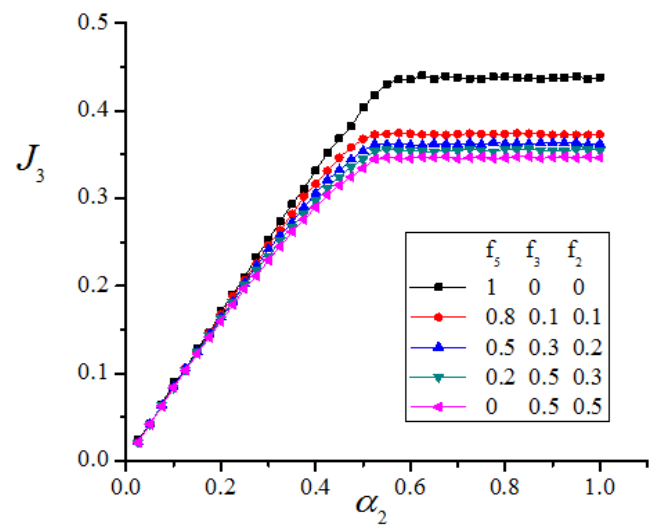

(b)

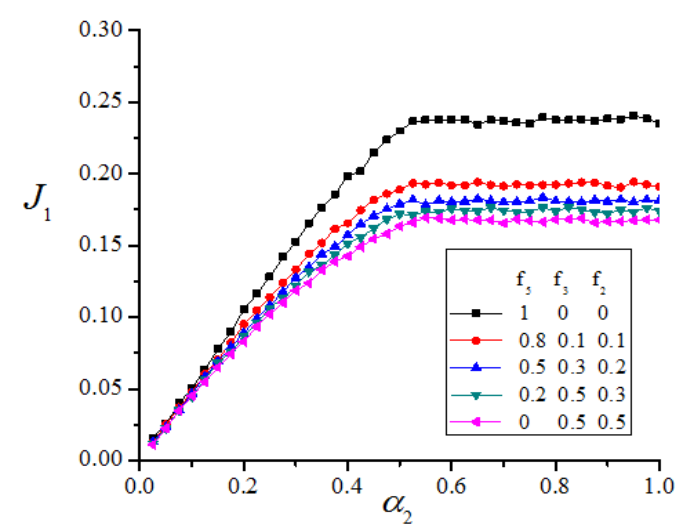

(c)

FIGURE III THE RELATIONS DIAGRAM BETWEEN MAIN LANE INJECTION PROBABILITY AND THE LANE TRAFFIC FLOW

\section{ACKNOWLEDGMENT}

The authors would like to gratefully acknowledge the support of Gansu Social Science Planning Program (Grants No.14YB051), Lanzhou Social Science Planning Program (Grants No.04-021E), Lanzhou Soft Science Research Program (Grants No.2013-3104).

\section{REFERENCES}

[1] Jia B, Gao Z Y, Li K P, et al, "Models and simulations of traffic system based on the theory of cellular automaton," Science Press, Beijing, 2007, pp. 58-112.

[2] Li X G, "Microscopic modeling and properties analyzing of transportion system based on cellular automata model," Beijing: Peking University, 2009, pp. 99-110.

[3] Wang S B, Qian Y S, Kang H X , "Cellular automaton model for mixing traffic in two-lane system with period boundary condition," Compute Application, vol. 28, 2008, pp. 2658-2660.

[4] Kuang H, Kong L J, Liu M R, "The study of a cellular automaton traffic flow model with mixed different maximum speed vehicles on single lane," Physica A, 2004, vol. 53, pp. 2894-2898.

[5] Zheng R S, Tan H L, Kong L J, et al, "A cellular automaton model for mixing traffic in two-lane system," Physica A, vol.54, 2005, pp. 35163523.Li Q L, Wang B H, Liu M R, "Phase diagrams properties of the mixed traffic on a crossroad," Physica A, vol.389, 2010, pp. 5045-5052.

[6] Tan H L, Zhang C Y, Kong L J, et al, "A study of the traffic flow on a crossroad of the mainroad," Journal of Guangxi Normal University, vol. 21, 2003, pp. 5-9.

[7] Xiao R J, Zhang L S, Tan H L, "Effects of mixed traffic flow at the crossroads," Physics of Guangxi, vol.29, 2008, pp. 25-28.

[8] Zhang X Q, Wang Y, Hu Q H, "Research and simulation on cellular automaton model of mixed traffic flow at intersection," vol.63, 2014, pp. 010508.1-010508.8.

[9] Li X G, Gao Z Y, Jia B, et al, "Cellular Automata Model for Unsignalized T-Shaped Intersection," International Journal of Modern Physics C, vol. 20, 2009, pp. 501-512.

[10] Fan H Q, Jia B, Li X G, et al, "Characteristics of traffic flow at nonsignalized T-shaped intersection with U-Turn movements," The Scientific World Journal, 2013, pp. 856416.1-856416.7. 